\title{
Acceleration of Relativistic Outflows with Tangled Magnetic Field
}

\author{
Shuta J. Tanaka* \\ Department of Physics and Mathematics, Aoyama Gakuin University, 5-10-1 Fuchinobe, \\ Sagamihara, Kanagawa 252-5258, Japan \\ E-mail: sjtanaka@phys.aoyama.ac.jp

\section{Kenji Toma} \\ Frontier Research Institute for Interdisciplinary Sciences, Tohoku University, Sendai 980-8578, \\ Japan \\ Astronomical Institute, Tohoku University, Sendai 980-8578, Japan
}

\begin{abstract}
Relativistic outflows are launched from compact stars, such as black holes and neutron stars. The flows are Poynting dominated at their origin because the rotational energy of the compact stars can be extracted by the magnetic braking mechanism. However, it is known that the efficient acceleration of the flows by converting the Poynting energy to kinetic one is not easy in the ideal magnetohydrodynamic (MHD) limit. Non-ideal MHD processes including dissipation of the magnetic field are discussed as an solution to the efficient flow acceleration. Here, we study the one-dimensional cylindrical relativistic outflow with non-ideal MHD effects. Radiative cooling (cooling effect), conversion of the ordered magnetic field into the turbulent one (conversion effect) and dissipation of the turbulent magnetic field (dissipation effect) are formulated according to our past study on pulsar wind nebulae. The cooling effect works as acceleration but the flow is not efficiently accelerated by the cooling effect alone. This is the same for the conversion effect. The dissipation effect rather works as deceleration. However, we find that the flow is efficiently accelerated to about a half of the maximum possible Lorentz factor when the cooling, conversion and dissipation effects work simultaneously. In this case, almost a half of the total luminosity of the flow is converted into the radiation.
\end{abstract}

High Energy Phenomena in Relativistic Outflows VII - HEPRO VII

9-12 July 2019

Facultat de Física, Universitat de Barcelona, Spain

\footnotetext{
* Speaker.
} 


\section{Introduction}

Relativistic outflows are associated with most of the observed high energy astrophysical phenomena. Extraction of the rotational energy of compact objects by magnetic braking has been discussed as a mechanism of driving the relativistic outflows, so that the outflow is Poynting dominated at its base. However, it is known that a Poynting dominated steady magnetohydrodynamic (MHD) flow in spherical symmetry is not efficiently accelerated in contrast to a thermally dominated relativistic fireball. Magnetic dissipation, which is a non-ideal MHD effect [1,2], is a candidate for acceleration mechanism of Poynting dominated flow.

Here, we study flow acceleration based on our past study of the pulsar wind nebula [3]. We apply the model to a cylindrical jet. Although the recent VLBI radio observations of the M87 jet reveal that the overall jet shape is parabolic rather than cylindrical $[4,5,6]$, the spines of the jets are expected to be of cylindrical shape [7,8]. Our formulation is a simple extension of the magnetic dissipation model $[1,2]$ and includes cooling of the plasma, conversion of the ordered magnetic field into the turbulent one and dissipation of the turbulent magnetic field (section 2). Radiative cooling is an essential property of the relativistic jets and recent numerical studies of relativistic jets also discuss the development of the turbulence and the magnetic dissipation inside the cylindrical jet $[8,9,10]$.

\section{Model}

Three non-ideal MHD terms are introduced from our past study: (1) cooling of plasma, (2) conversion of the ordered toroidal magnetic field into the turbulent one, and (3) dissipation of the turbulent magnetic field into plasma heat. For simplicity, we assume that the flow is (i) steady, (ii) has (cylindrical) one-dimension along the jet axis $z$, (iii) a velocity field along the jet axis (no transverse component) and (iv) no lateral structure. For the magnetic field, (v) transverse (toroidal) and turbulent components are considered. The system has six variables: the proper enthalpy density $w$, the pressure $p$, the proper mass density $\rho$, the four velocity along the jet axis $u \equiv \gamma \beta$, the strength of the ordered transverse magnetic field $\bar{b}$ in the proper frame and the strength of the turbulent magnetic field $\delta b$ which is isotropic in the proper frame $\left(\langle\delta \boldsymbol{b}\rangle=0\right.$ and $\left.\left\langle\delta \boldsymbol{b}^{2}\right\rangle=\delta b^{2}\right)$.

The equation of state of a Synge gas $[11,12]$ is adopted,

$$
w(z)=\omega(\Theta) \rho c^{2}, p(z)=\Theta \rho c^{2}, \omega(\Theta) \equiv \frac{K_{3}\left(\Theta^{-1}\right)}{K_{2}\left(\Theta^{-1}\right)},
$$

where the non-dimensional temperature $\Theta(z)$ is normalized by the rest mass energy of the plasma particles, $K_{V}(x)$ is the modified Bessel function of the second kind and $\omega(\Theta) \rightarrow 1$ for $\Theta \rightarrow 0$. The effective adiabatic index of the Synge gas is $\hat{\Gamma}(z) \equiv \omega^{\prime}(\Theta) /\left(\omega^{\prime}(\Theta)-1\right)$, where the dash ' denotes a derivative with respect to $\Theta$. Equation of continuity is integrable and we obtain the conservation of the mass flux

$$
\dot{m} \equiv \rho(z) u(z) c=\text { const. }
$$


Other flow variables are determined from the four equations,

$$
\begin{aligned}
\frac{d}{d z}\left[\gamma u\left(w+\bar{b}^{2}+\frac{2}{3} \delta b^{2}\right)\right] & =-\gamma \frac{\Lambda_{\mathrm{cool}}}{c}, \\
\frac{d}{d z}(w-p)+w \frac{d}{d z} \ln u & =\frac{\delta b^{2} / 2}{u c \tau_{\mathrm{diss}}}-\frac{\Lambda_{\mathrm{cool}}}{u c}, \\
\frac{d}{d z} \frac{\bar{b}^{2}}{2}+\frac{\bar{b}^{2}}{2} \frac{d}{d z} \ln u^{2} & =-\frac{\bar{b}^{2} / 2}{u c \tau_{\mathrm{conv}}}, \\
\frac{d}{d z} \frac{\delta b^{2}}{2}+\frac{2}{3} \delta b^{2} \frac{d}{d z} \ln u & =-\frac{\delta b^{2} / 2}{u c \tau_{\mathrm{diss}}}+\frac{\bar{b}^{2} / 2}{u c \tau_{\mathrm{conv}}},
\end{aligned}
$$

which represent the conservation laws of the total energy flux, the plasma internal energy, and the energies of the ordered and turbulent magnetic field, respectively. All the non-ideal MHD effects are on the right-hand side of equations $(2.3)-(2.6)$, where $\Lambda_{\text {cool }}, \tau_{\text {conv }}$ and $\tau_{\text {diss }}$ have positive values and are parameters of the system characterizing the cooling, conversion and dissipation effects, respectively.

le introduce the total energy density $\varepsilon(z)^{1}$, the total energy flux $l(z)$, the magnetization $\sigma(z)$, and the characteristic velocity $\beta_{\mathrm{c}}(z)$ as

$$
\begin{aligned}
\varepsilon(z) & \equiv w+\bar{b}^{2}+(2 / 3) \delta b^{2}, \\
l(z) & \equiv \gamma u c \varepsilon, \\
\sigma(z) & \equiv \frac{\bar{b}^{2}+(2 / 3) \delta b^{2}}{w}, \\
\beta_{\mathrm{c}}^{2}(z) & \equiv \frac{\hat{\Gamma} p+\bar{b}^{2}+(2 / 9) \delta b^{2}}{\varepsilon} .
\end{aligned}
$$

The characteristic velocity $\beta_{\mathrm{c}}$ corresponds to the fast magnetosonic velocity when $\delta b=0$. Combining equations $(2.3)-(2.6)$ with the use of the above quantities, we obtain the differential equation for the four velocity

$$
\varepsilon\left(\beta^{2}-\beta_{\mathrm{c}}^{2}\right) \frac{d u}{d z}=(\hat{\Gamma}-1) \frac{\Lambda_{\mathrm{cool}}}{c}+\frac{\bar{b}^{2}}{3 c \tau_{\mathrm{conv}}}+\left(\frac{4}{3}-\hat{\Gamma}\right) \frac{\delta b^{2}}{2 c \tau_{\mathrm{diss}}}
$$

Below, we consider the flow of $\beta>\beta_{\mathrm{c}}>0$ and then the sign of the right-hand side of eqution (2.12) equals to that of $d u / d z$, i.e., the flow is accelerated along the jet axis $z$ when the sign of the right-hand side of equation (2.12) is positive. The first two terms of the right-hand side of equation (2.12) are always positive because the effective adiabatic index $\hat{\Gamma}$ ranges from $4 / 3$ (ultrarelativistic temperature $\Theta \gg 1$ ) to $5 / 3$ (non-relativistic temperature $\Theta \ll 1$ ) for a Synge gas. The magnetic dissipation term in equation (2.12) is the only negative (deceleration) term. We will see that deceleration by magnetic dissipation is inefficient because magnetic dissipation simultaneously heats up the plasma $\hat{\Gamma} \rightarrow 4 / 3$ and the third term of the right-hand side of equation (2.12) tends to zero. Finally, we note that equation (2.12) does not have the (geometrical) 'expansion acceleration'

\footnotetext{
${ }^{1}$ It might be better to call $\varepsilon(z)$ the total enthalpy density because the value defined by equation (2.7) is the sum of the enthalpy density of the plsama, the toroidal and the turbulent magnetic field.
} 
term which is the first term of the right-hand side of the equation (19) of [3], and is proportional to $p / r$. The spherical geometry is adoted by [3] for stuyding pulsar wind nebulae, whilei, here, the cylindrical geometry is adopted for studying the relativistic jets.

Five boundary conditions are set at an inlet boundary $z=z_{0}$, where $x \equiv z / z_{0}$ below. The energy density at the boundary (I) $l_{0} / c \equiv \gamma_{0} u_{0} \omega\left(\Theta_{0}\right) \rho_{0} c^{2}\left(1+\sigma_{0}\right)=1$, the maximum attainable Lorentz factor (II) $\gamma_{\max } \equiv l_{0} /\left(\dot{m} c^{2}\right)=10^{4}$, and the inlet turbulent magnetic field (III) $\delta b_{0}^{2}=0$ are fixed. The magnetization of the flow is an important parameter and we study (IV) $\sigma_{0}=10^{-3}, 1,10,10^{2}$. The inlet four velocity is set to be $(\mathrm{V}) u_{0}=u_{\mathrm{c}, 0}$, where

$$
u_{\mathrm{c}, 0}^{2}=\frac{\left(\hat{\Gamma}-1+\sigma_{0}\right) \gamma_{\max }-(\hat{\Gamma}-1)\left(1+\sigma_{0}\right) \gamma_{0}}{(2-\hat{\Gamma}) \gamma_{\max }+(\hat{\Gamma}-1)\left(1+\sigma_{0}\right) \gamma_{0}} .
$$

In practice, the inlet velocity $u_{0}$ is infinitesimally larger than $u_{\mathrm{c}, 0}$, i.e., we have acceleration solutons because the right-hand side of equation (2.12) is positive. Note that the inlet boundary conditions (I) and (II) gives a physical constraint $\gamma_{\max } \geq \gamma_{0}\left(1+\sigma_{0}\right)$ for $\Theta_{0} \geq 0\left(\omega\left(\Theta_{0}\right) \geq 0\right)$. The constraint gives an upper limit of $\sigma_{0}$ because $\gamma_{0}=\gamma_{\mathrm{c}} \sim \sqrt{\sigma_{0}}$ for $\sigma_{0} \gg 1$, i.e., $\sigma_{0} \lesssim \gamma_{\max }^{2 / 3}$.

The non-ideal MHD effects are parameterized by corresponding three length-scales. Definitions of the cooling, conversion and dissipation lengths are $x_{i} \equiv c \tau_{i} / z_{0}(i=$ cool, conv, and diss). $\tau_{\text {conv }}$ and $\tau_{\text {diss }}$ are already introduced in equtions (2.4) - (2.6). For $\tau_{\text {cool }}$, we assume the form of the cooling rate $\Lambda_{\text {cool }} \equiv e_{\text {int }} / \tau_{\text {cool }}$, where $e_{\text {int }}=w-p-\rho c^{2}=(\omega(\Theta)-\Theta-1) \rho c^{2}$ is the internal energy density of plasma [13]. The unit $c=1$ is adopted below.

\section{Results}

Figure 1 shows the resultant flow profiles, where we calculate the flow from $x_{0}=1$ out to $x_{\text {out }}=10^{10}$. From top to bottom, the four velocity, magnetization, and energy flux profiles are shown. From left to right, the adopted values of the characteristic length-scales are different. For each panels, the color of lines represents the inlet magnetization $\sigma_{0}=10^{-3}$ (black), $\sigma_{0}=1$ (red), $\sigma_{0}=10$ (blue), and $\sigma_{0}=10^{2}$ (green), respectively.

At first, we studied each of the cooling, conversion and dissipation effects for $10 \leq x_{i} \leq 10^{9}$ ( $i=$ cool, conv, and diss). The cooling (section 3.1) and conversion (section 3.2) effects work as acceleration, while the dissipation effect alone does not change the flow profiles because $\delta b$ is always zero without the conversion effect. In section 3.3, we presents interesting cases that all the three non-ideal MHD effects works. The adopted parameters and the derived terminal values for each result are summarized in Table 1.

\subsection{Cooling acceleration}

The left three panels of Figure 1 are the results of cooling acceleration, which is important for a relativistically-hot flow. The inlet temperature is $\Theta_{0} \approx 2.0 \times 10^{3}$ for $\sigma_{0}=10^{-3}$, while $\Theta_{0} \approx 2.0$ for $\sigma_{0}=10^{2}$. The conversion and dissipation length-scales are set to infinity and then the system has the only one length-scale $x_{\text {cool }}$. The results for different $x_{\text {cool }}$ have similarity curves, i.e., $x_{\text {cool }}$ can be chosen as the normalization of $z_{0}$ and the terminal values $u_{\infty}, \sigma_{\infty}, l_{\infty}$ are the same for different $x_{\text {cool }}$. Here, we only shows the results for $x_{\text {cool }}=10^{3}$. The adopted parameters and the derived terminal values are summarized in Table 1 . 


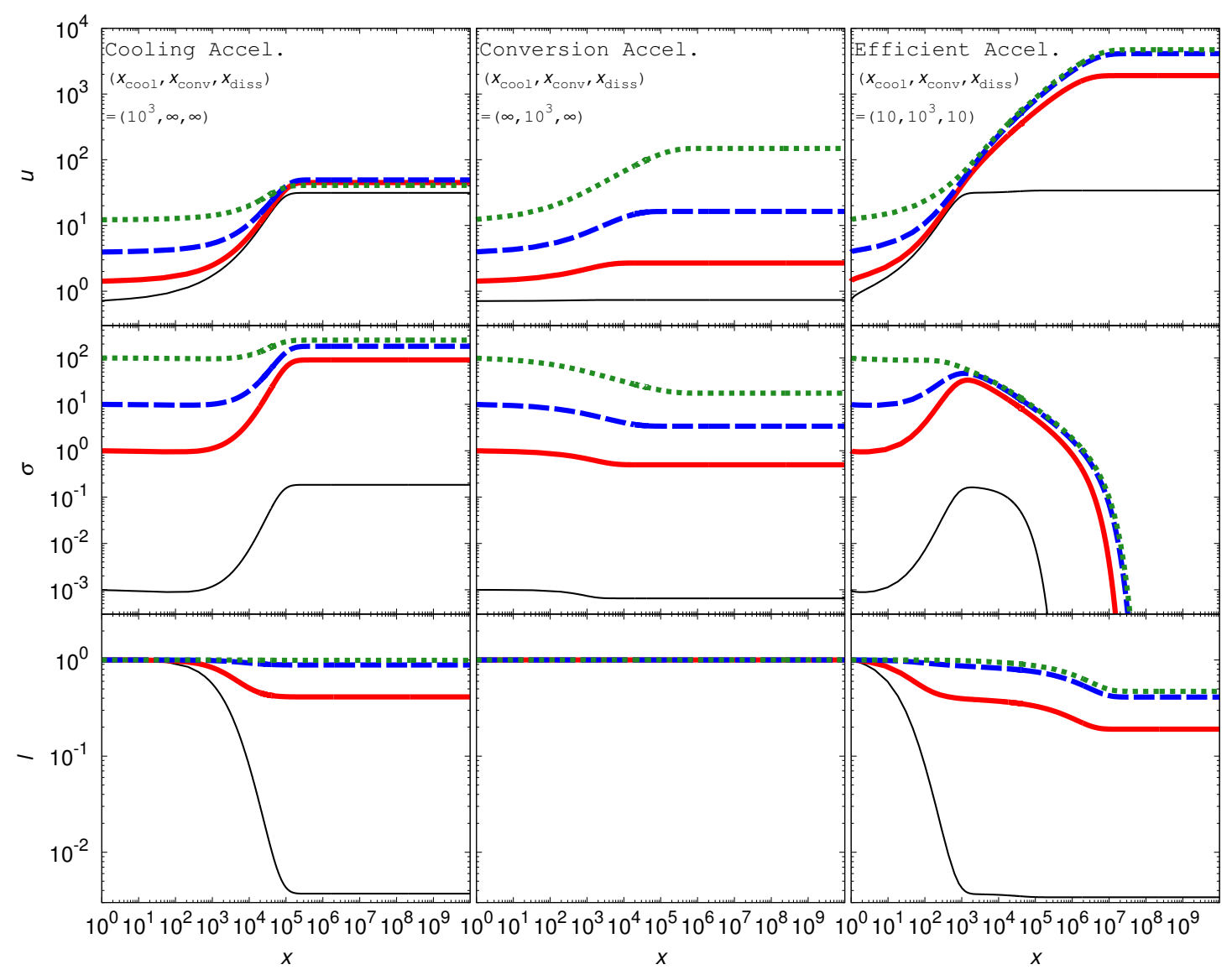

Figure 1: The profiles of the four velocity $u(x)$ (top row), the magnetization $\sigma(x)$ (middle row) and the total energy flux $l(x)$ (bottom row). The line color is different for the different inlet boundary conditions $\sigma_{0}=10^{-3}$ (black line), 1 (red line), 10 (blue dashed line), and $10^{2}$ (green dotted line). For the left panels (cooling acceleration), the conversion and dissipation effects are ignored $\left(x_{\text {conv }}=x_{\text {diss }}=\infty\right)$. For the center panels (conversion acceleration), the cooling and dissipation effects are ignored $\left(x_{\text {cool }}=x_{\text {diss }}=\infty\right)$. For the right panels (efficient acceleration), all the non-ideal MHD effects are included ( $x_{\text {cool }}=x_{\text {diss }}=10$ and $x_{\text {conv }}=10^{3}$ ). The adopted parameters are summarized in Table 1.

The terminal four velocity is less than fifty for all the cases and is much smaller than $\gamma_{\max }$. The flows start to accelerate at $x \approx x_{\text {cool }}$ and the four velocity profiles obey $u \propto x^{3 / 4}$ in the acceleration phase. The terminal magnetization also increases at $x \approx x_{\text {cool }}$ because of the flow compression. The terminal energy flux is much less than unity for $\sigma_{0}=10^{-3}$, i.e., $\approx 99.6 \%$ of the jet power is taken away from the system by radiative cooling.

\subsection{Conversion acceleration}

The middle three panels of Figure 1 are the results of conversion acceleration, which is important for the high- $\sigma_{0}$ flow. The cooling and dissipation effects are omitted for all the curves in the middle three panels. The system has the only one length-scale $x_{\text {conv }}$. The results are similar and the 
Table 1: Summary of the inlet boundary conditions $\left(\sigma_{0}\right)$, the characteristic length-scales $\left(x_{\text {cool }}, x_{\text {conv }}, x_{\text {diss }}\right)$ and the derived terminal values $\left(u_{\infty}, \sigma_{\infty}, l_{\infty}\right)$ for Fig. 1.

\begin{tabular}{cccc|ccc}
\hline \multicolumn{6}{c}{ Adopted } \\
\hline \multicolumn{7}{c}{ Cooling Acceleration } \\
\hline$\sigma_{0}$ & $x_{\text {cool }}$ & $x_{\text {conv }}$ & $x_{\text {diss }}$ & $u_{\infty}$ & $\sigma_{\infty}$ & $l_{\infty}$ \\
\hline $10^{2}$ & $10^{3}$ & $\infty$ & $\infty$ & 41 & 240 & 0.99 \\
$10^{1}$ & $10^{3}$ & $\infty$ & $\infty$ & 49 & 180 & 0.89 \\
$10^{0}$ & $10^{3}$ & $\infty$ & $\infty$ & 45 & 90 & 0.41 \\
$10^{-3}$ & $10^{3}$ & $\infty$ & $\infty$ & 31 & 0.18 & 0.0037 \\
\hline \multicolumn{6}{c}{ Conversion Acceleration } \\
\hline$\sigma_{0}$ & $x_{\text {cool }}$ & $x_{\text {conv }}$ & $x_{\text {diss }}$ & $u_{\infty}$ & $\sigma_{\infty}$ & $l_{\infty}$ \\
\hline $10^{2}$ & $\infty$ & $10^{3}$ & $\infty$ & 150 & 17 & 1 \\
$10^{1}$ & $\infty$ & $10^{3}$ & $\infty$ & 16 & 3.4 & 1 \\
$10^{0}$ & $\infty$ & $10^{3}$ & $\infty$ & 2.7 & 0.50 & 1 \\
$10^{-3}$ & $\infty$ & $10^{3}$ & $\infty$ & 0.74 & 0.00066 & 1 \\
\hline \multicolumn{7}{c}{ Efficient Acceleration } \\
\hline$\sigma_{0}$ & $x_{\text {cool }}$ & $x_{\text {conv }}$ & $x_{\text {diss }}$ & $u_{\infty}$ & $\sigma_{\infty}$ & $l_{\infty}$ \\
\hline $10^{2}$ & 10 & $10^{3}$ & 10 & 4700 & 0 & 0.47 \\
$10^{1}$ & 10 & $10^{3}$ & 10 & 4100 & 0 & 0.41 \\
$10^{0}$ & 10 & $10^{3}$ & 10 & 1900 & 0 & 0.19 \\
$10^{-3}$ & 10 & $10^{3}$ & 10 & 34 & 0 & 0.0034
\end{tabular}

terminal values $u_{\infty}, \sigma_{\infty}, l_{\infty}$ are the same for different $x_{\text {conv }}$. The adopted parameters and the derived terminal values are summarized in Table 1 .

The terminal four velocity increases with $\sigma_{0}$ while is also much smaller than $\gamma_{\max }$. Note that the inlet magnetization has the maximum value $\sigma_{0} \lesssim \gamma_{\max }^{2 / 3} \approx 460$ for $\gamma_{\max }=10^{4}$ from the requirement $\Theta_{0}>0$. The flows start to accelerate at $x \approx x_{\text {conv }}$ and the four velocity profiles obey $u \propto x^{1 / 3}$ in the acceleration phase. The terminal magnetization also decreases at $x \approx x_{\text {cool }}$ because the conversion of the magnetic configuration from the toroidal to turbulent one reduces the magnetization. The terminal energy flux is constant because of no cooling effect.

\subsection{Efficient acceleration}

The right three panels of Figure 1 are the results of efficient acceleration $\left(u_{\infty} \sim \gamma_{\max }\right)$. All the non-ideal MHD effects are considered for efficient acceleration $\left(x_{\text {cool }}, x_{\text {conv }}, x_{\text {diss }}\right)=\left(10,10^{3}, 10\right)$. Note that both cooling and conversion acceleration work even for the other combinations of the characteristic length-scales but the flows are not always accelerated efficiently. We find that $x_{\text {conv }} \gg$ $x_{\text {cool }}, x_{\text {diss }}$ are required for efficient acceleration. The adopted parameters and the derived terminal values are summarized in Table 1.

The terminal four velocity increases with $\sigma_{0}$ and attains $\approx \gamma_{\max } / 2$ for the high- $\sigma_{0}$ cases. In addition to conversion acceleration, dissipation of the turbulent magnetic field into plasma heat allows cooling acceleration because the flow is much hotter than the cases without magnetic dissipation. 
The terminal magnetization is zero because of magnetic conversion and dissipation. The terminal energy flux is larger for larger $\sigma_{0}$, i.e., for larger $u_{\infty}$.

\section{Discussion}

Acceleration of cylindrical jets is studied by including the three non-ideal MHD effects, namely the cooling $x_{\text {cool }}$, conversion $x_{\text {conv }}$, and dissipation effects $x_{\text {diss }}$ [3]. Our non-ideal MHD formulation is heuristic but would be a way to fill the gap between simplified analytic (ideal MHD) studies and more realistic numerical studies. There are some other non-ideal MHD terms which make both analytical and numerical (theoretical) studies more realistic. For example, mass loading to the jet $[14,15]$ and effects of multi-composition in the plasma [16].

\section{Acknowledgements}

S. J. T. would like to thank anonymous referee for his/her careful reading of the manuscript. This work is supported by JSPS Grants-in-Aid for Scientific Research Nos. 17H18270 (SJT) and $18 \mathrm{H} 01245$ (KT).

\section{References}

[1] Y. Lyubarsky and J. G. Kirk, Reconnection in a Striped Pulsar Wind, ApJ 547 (2001) 437 [astro-ph/0009270].

[2] G. Drenkhahn, Acceleration of GRB outflows by Poynting flux dissipation, A\&A 387 (2002) 714 [astro-ph/0112509].

[3] S. J. Tanaka, K. Toma and N. Tominaga, Confinement of the Crab Nebula with tangled magnetic field by its supernova remnant, MNRAS 478 (2018) 4622 [1805. 08325].

[4] K. Asada and M. Nakamura, The Structure of the M87 Jet: A Transition from Parabolic to Conical Streamlines, ApJl 745 (2012) L28 [1110 . 1793].

[5] K. Hada, M. Kino, A. Doi, H. Nagai, M. Honma, Y. Hagiwara et al., The Innermost Collimation Structure of the M87 Jet Down to 10 Schwarzschild Radii, ApJ 775 (2013) 70 [1308.1411].

[6] M. Nakamura, K. Asada, K. Hada, H.-Y. Pu, S. Noble, C. Tseng et al., Parabolic Jets from the Spinning Black Hole in M87, ApJ 868 (2018) 146 [1810 . 09963].

[7] V. S. Beskin and E. E. Nokhrina, On the central core in MHD winds and jets, MNRAS 397 (2009) $1486[0810.4307]$.

[8] O. Porth and S. S. Komissarov, Causality and stability of cosmic jets, MNRAS 452 (2015) 1089 [1408.3318].

[9] O. Bromberg and A. Tchekhovskoy, Relativistic MHD simulations of core-collapse GRB jets: $3 D$ instabilities and magnetic dissipation, MNRAS 456 (2016) 1739 [1508.02721].

[10] O. Bromberg, C. B. Singh, J. Davelaar and A. A. Philippov, Kink instability: evolution and energy dissipation in Relativistic Force-Free Non-Rotating Jets, arXiv e-prints (2019) arXiv:1908.08620 [1908.08620].

[11] F. Jüttner, Die relativistische Quantentheorie des idealen Gases, Zeitschrift fur Physik 47 (1928) 542. 
[12] J. L. Synge, The Relativistic Gas. North-Holland, 1957.

[13] G. Drenkhahn and H. C. Spruit, Efficient acceleration and radiation in Poynting flux powered GRB outflows, A\&A 391 (2002) 1141 [astro-ph/ 0202387 ].

[14] S. S. Komissarov, Mass-Loaded Relativistic Jets, MNRAS 269 (1994) 394.

[15] K. Toma and F. Takahara, Baryon Loading of Active Galactic Nucleus Jets Mediated by Neutrons, ApJ 754 (2012) 148 [1205.6868].

[16] U. Motschmann, K. Sauer and T. Roatsch, simulation of ion acceleration in a charged dust cloud, Geophys. Res. Lett. 19 (1992) 225. 